\title{
Macrophage supernatant infected with Leishmania major mediates the cytology of fibroblast cells in skin wounds
}

\author{
Mohsen Ghomashlooyan, ${ }^{1}$ Fatemeh Namdar, ${ }^{1}$ Mehrafarin Fesharaki, ${ }^{2}$ \\ Shervin Ghaffari Hosseini, ${ }^{3}$ Manizheh Narimani, ${ }^{1}$ Zahra Sebghatollahi, ${ }^{4}$ Bahareh Basirpour, ${ }^{1}$ \\ Seyed Hossein Hejazi ${ }^{4}$ \\ ${ }^{1}$ Department of Parasitology and Mycology, School of Medicine; ${ }^{2}$ Department of Physiology, School of \\ Medicine; ${ }^{3}$ Cardiac Rehabilitation Research Center, Cardiovascular Research Institute, Isfahan University \\ of Medical Sciences, Isfahan; ${ }^{4}$ Skin Diseases and Leishmaniasis Research Center, Department of \\ Parasitology and Mycology, School of Medicine, Isfahan University of Medical Sciences, Isfahan, Iran
}

\begin{abstract}
Objective: Healing of Cutaneous Leishmaniasis relies on the effective and modulates protective immune responses. Although the immune system is necessary to eliminate the parasite, it could be considered as the main cause of ulcers. Therefore, main aim of this study was to explore the possible regulatory functions of macrophage supernatant infected with Leishmania major on the fibroblast cells.

Materials and Methods: In this experimental study, different concentrations of infected macrophage supernatant extract (50, $100,150,200$, and $250 \mu \mathrm{g} / \mathrm{mL})$ were tested at different times $(6,24$, 48 , and $72 \mathrm{~h}$ ) and the effect of the leishmanicidal extract on fibroblast cells was determined by MTS assay. Also, the flow-cytometry technique was used for the investigation of apoptosis induction percentage.
\end{abstract}

\footnotetext{
Correspondence: Seyed Hossein Hejazi, Skin Diseases and Leishmaniasis Research Center, Department of Parasitology and Mycology, School of Medicine, Isfahan University of Medical Sciences, Isfahan, Iran. Tel.: +98 9133118711

E-mail: hejazi@med.mui.ac.ir
}

Key words: Fibroblast cell; Leishmania major; apoptosis; flow cytometry; MTS assay.

Conflict of interest: The authors declare no conflict of interest.

Informed consent: For this type of study, formal consent is not required.

Ethics approval: IR.MUI.REC. 394249

Availability of data and materials: All data generated or analyzed during this study are included in this published article.

Received for publication: 29 May 2020.

Revision received: 12 October 2020.

Accepted for publication: 12 October 2020 .

This work is licensed under a Creative Commons Attribution NonCommercial 4.0 License (CC BY-NC 4.0).

CC Copyright: the Author(s),2020

Licensee PAGEPress, Italy

Infectious Diseases and Herbal Medicine 2020; 1:83

doi:10.4081/idhm.2020.83
Results: MTS assay showed that the leishmanicidal effect of infected macrophage supernatant extract was dependent on the concentration and the time of treatment. So, the best efficacy was observed in $200 \mu \mathrm{g} / \mathrm{mL}$ with 72 hours exposure time. Flow cytometry analysis showed that the infected macrophage supernatant extract could induce apoptosis in cultured fibroblasts.

Conclusions: We have demonstrated that reduction of survival rate and induction of apoptosis in fibroblasts displayed a similar manner to keratinocytes when exposed to infected macrophages with L. major. Our data suggest that such a phenomenon can be the underlying cause of lesions with scarring, and future, the mechanism remains to be elucidated.

\section{Introduction}

Leishmaniasis is a disease caused by obligate and intracellular parasites belonging to the Leishmania genus. Cutaneous Leishmaniasis (CL) is caused by the inoculation of the parasite Leishmania from sandflies to humans. The severity of the disease depends on the species and inducing host immune responses. Several preventive efforts, such as insect vector control and vaccination, were performed to control the disease, but none of them had been entirely successful. ${ }^{1,2}$ Both wound healing and the creation process are depended on the host immune response. Although the immune system is necessary to eliminate the parasite, it could be considered as the leading cause of ulcers, as demonstrated by the absence of ulcers in patients with AIDS. ${ }^{3}$ The presence of parasites in wound site after years of wound healing indicates that wound healing is not only related to the parasites elimination and the presence of the parasites is not the only factor playing a role in the establishment of such wounds. Thus, it seems that how the host immune system response to the parasite leads to scarring.

Since Leishmania major (L. major) is an intracellular parasite able to survive and proliferate only in specific immune system cells such as macrophages in vertebrate hosts, its interaction with adjacent cells is possible only by changing the behavior of the immune cells. ${ }^{4-6}$ In many skin lesions, activation of the immune system (by autoimmune or external antigen) would induce cell death in cells of the epidermis by apoptosis, and which is one of the most important causes of skin ulcers. ${ }^{7-9}$ In a few studies which investigate the mechanism of injury caused by Leishmaniasis, it has been demonstrated that L. major leads to induce apoptosis in the keratinocytes. ${ }^{10-14}$ However, to create wounds that scars, epidermis cells death are not enough solely, and dermis natural building also should be significantly damaged to remain a healing scar. ${ }^{15}$ So infected macrophage and sensitized lymphocytes with $L$. 
major not only effect on epidermal cell apoptosis but also have a significant impact on dermis structure and cells.

As mentioned, the behaviors of the dermis and epidermis cells are unknown in response to Leishmania infection, and the effect on tissue regeneration and wound healing is still unclear. Even there is no complete information in the case of loss of cells (apoptosis, necrosis, or reduce proliferation). Therefore, in this study, the authors are supposed to understand the necessary information about the effect of Leishmania on the most important cells of the epidermis and dermis, the keratinocytes and fibroblasts. However, the epidermis cell death has been explicitly investigated by molecular mechanisms in previous studies. ${ }^{16-18}$ However, transfected cell lines or only one normal cell line was used in previous works cannot be a true representative of the epidermis cells. Therefore, in this study, we are looking for more reliable picture from stimulation of macrophage with $L$. major and their effect on human fibroblast by using cells from donors and with the least possible passage in the culture medium. On the other hand, primary information about the effects of L. major on the skin tissues was also achieved by fibroblast cells culture and compared to the effectiveness of parasitic infection on fibroblasts and keratinocytes.

\section{Materials and Methods}

\section{Parasite Culture}

The strain of L. major (MRHO/IR/75/ER) was obtained in cryopreserved from Department of Parasitology and Mycology, School of Medicine, Isfahan University of Medical Sciences, Isfahan, Iran. Promastigotes were cultured in RPMI 1640 (Gibco, UK) enriched with FBS 10\% (Fetal Bovine Serum, Bio-idea, Iran), $100 \mathrm{IU} / \mathrm{mL}$ of penicillin, and $100 \mu \mathrm{g} / \mathrm{mL}$ of streptomycin and 100 $\mu \mathrm{g} / \mathrm{mL}$ gentamycin for mass production. ${ }^{19}$

\section{Isolation of macrophage by Ficoll density gradient separation}

The Ficoll density gradient separation of whole blood remains the most commonly used procedure for the separation of mononuclear cells. First, $20 \mathrm{~mL}$ of blood from the collection vial was transferred to a $50 \mathrm{~mL}$ tube, and an equal volume of PBS was added and mixed. $15 \mathrm{~mL}$ Ficoll (Biosera, England) was added in a second $50 \mathrm{~mL}$ tube and carefully layered the diluted blood over the Ficoll. In the following, the tube was centrifuged without the stop at $400 \mathrm{~g}$ for $30 \mathrm{~min}$ at $18-24^{\circ} \mathrm{C}$. The tubes were carefully removed from the centrifuge so as not to disturb the layering and remove the macrophage layer and transfer to a new $50 \mathrm{~mL}$ tube. In the next step, we washed macrophage fraction by PBS and centrifuged it at $100 \mathrm{~g}$ for $10 \mathrm{~min}$ at $18-24^{\circ} \mathrm{C}$ in triplicate. Finally, the supernatant was decanted, and the cell pellet was re-suspended by the appropriate volume of PBS (or media). The cells were cultured in RPMI 1640 medium (Bio-idea, Iran) supplemented with $2 \mathrm{mM}$ L-glutamine, $100 \mathrm{U} / \mathrm{mL}$ penicillin, $100 \mu \mathrm{g} / \mathrm{mL}$ streptomycin, $10 \%$ heatinactivated FBS, which was changed on day 2 and every 3 days subsequently. Macrophages were used between days 7 and 10 of culture. ${ }^{20}$ LPS is known to induce the appearance of cell characteristics consistent with mature macrophages. ${ }^{21}$

\section{L. major Infected Macrophages}

In the next step, L. major was added to cultured cells in a ratio of ten parasites to one cell and was incubated at $37^{\circ} \mathrm{C}$ in complete RPMI 1640 medium. After 24 hours, the monolayers were extensively washed to remove extracellular parasites and adherent cells.
The supernatant was obtained in 24, 48, 72, and 96 hours after incubation and was frozen at $-20^{\circ} \mathrm{C} .^{22}$

\section{Fibroblast cell culture}

The fibroblast cells were obtained by a skin punch, Iran. Cells were cultured in RPMI 1640 medium (Gibco-Invitrogen) supplied by $10 \%$ fetal bovine serum (Gibco-Invitrogen), $1 \%$ antibiotic mixture containing penicillin (Sigma-Aldrich) and streptomycin (Sigma-Aldrich). The cells were stored at a humidified atmosphere at $37{ }^{\circ} \mathrm{C}$ with $5 \% \mathrm{CO}_{2}$. The culture medium of cells was changed approximately every two days, and when they reached more than $80 \%$ confluence, they were split with $0.05 \%$ Trypsin $/ 0.02 \%$ EDTA and sub-cultured for more passages.

\section{Cell proliferation measurements by colorimetric MTS assay}

Cells were seeded at $4 \times 10^{3}$ per well of a 96 -well tissue culture plate. After 48 hours, the supernatant of infected macrophages and uninfected macrophages $(24,48,72$, and $96 \mathrm{~h})$ were removed by centrifugation and then added to the fibroblast cells. Cell viability was investigated using the MTS assay, the Cell Titer 96 Aqueous One Solution Cell Proliferation (ProMega), after 48 hours. Optical Density (OD) was recorded at $490 \mathrm{~nm}$ in a 96 -well plate reader (Biorad). Cell survival was evaluated using the following equation: ${ }^{23,24}$

Survival fraction $=\frac{\text { mean OD in test wells }- \text { mean OD in cell free wells }}{\text { mean OD in control wells }- \text { mean OD in cell free wells }}$

\section{Apoptosis assessment with flow cytometry}

Apoptotic cells were detected using PI staining of small DNA fragments followed by flow cytometry. The Annexin-V Staining Kit (Roche, Germany) with Propidium Iodide (PI) was used for the detection of apoptotic and necrotic cells according to the manufacturers protocol. Briefly, the wells were treated with $20 \mathrm{mg} / \mathrm{mL}$ concentration of extract and were incubated at $24{ }^{\circ} \mathrm{C}$. After 72 hours, promastigotes were washed in cold Phosphate-Buffered Saline (PBS) and centrifuged at $1400 \mathrm{~g}$ for $10 \mathrm{~min}$ and the pellet re-suspended in the binding buffer to a concentration of $1 \times 105 / \mathrm{mL}$ of promastigotes. Afterward, they were incubated for $15 \mathrm{~min}$ at the room temperature in darkness, with $10 \mu \mathrm{L}$ of Annexin-V in the presence of PI. Then, the samples were analyzed with FACS Calibur flow cytometer (Becton Dickinson and Cell Quest software), and the percentage of positive cells was determined for each sample. ${ }^{24,25}$

\section{Statistical analysis}

The data were presented as the mean \pm SD. Finally, the data were analyzed using SPSS for Windows (Version 16.0) (SPSS Inc., Chicago, IL, USA), and $\mathrm{P}<0.05$ was considered a significant level.

\section{Results}

\section{A decrease of fibroblast cell survival fraction by infected macrophage}

The cells were incubated with 24, 48, 72, and 96 hours-supernatant. As shown in Figure 1, no significant differences were seen between the controls and those treated with the 24 and 48 hourssupernatant over the whole range of both supernatant concentrations $(p>0.05)$. Survival fraction decreased after incubation with 
72 and 96 hours-supernatant so that this reduction was more considerable for 96 hours over the whole range of both supernatant concentrations $(p<0.05)$. These results indicated that 96 hourssupernatant had remarkable cytotoxicity on fibroblast cells. Therefore, it was selected as a useful supernatant. Also, there was no significant difference between the survival fraction of cells incubated with infected and normal macrophage ( $p$-value $>0.05$ )

\section{Infected macrophage cause fibroblast cell apoptosis}

To evaluate apoptosis, dual staining was used. Annexin VFITC represents early apoptosis (lower right quadrant, LR) for

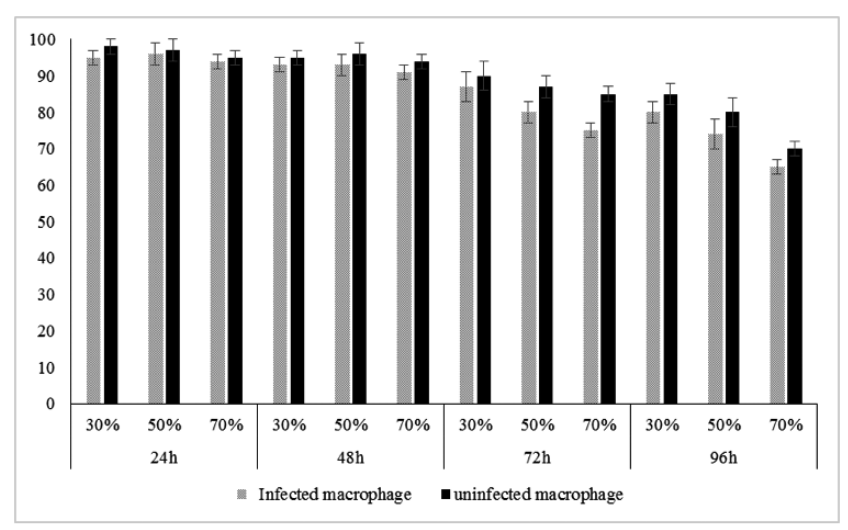

Figure 1. Survival fraction of fibroblast cells after incubation with $24,48,72$ and 96 hours-supernatant. internalized Phosphatidylserine (PS), and PI is the symbol of late apoptosis (upper right quadrant, UR). Therefore, the sum of LR and UR represents the apoptosis rate. Figure 2 shows the fraction of fibroblast cells undergoing apoptosis 48 hours after incubation with 96 hours-supernatant. Significant increase apoptosis was observed in fibroblast cells, which incubated with 96h-supernatant compared to control cells $(\mathrm{p}<0.05)$.

\section{Discussion}

Macrophages have long been considered to be crucial immune effector cells. They also play an important role in purifying the cells destroyed by apoptosis and necrosis. Phagocytosis of these components by macrophages leads to dramatic changes in their physiology, including alterations in the expression of surface proteins and the production of cytokines and pro-inflammatory mediators. ${ }^{26}$ Both cutaneous wound creation and healing processes are depended on the host immune response to the parasite. Although the immune response is required to remove parasites, it can be a significant cause of ulcers. The main objective of this study was to investigate the released from macrophages after infection with $L$. major and their effects on fibroblast as the central constructive cells of the skin. ${ }^{27}$

Based on our findings, when fibroblasts were exposed to macrophages infected with Leishmania major, the reduction of survival rate and induction of apoptosis occurred similarly to keratinocytes through secreting various elements that are more or less known. This phenomenon can be the underlying cause of lesions with scarring. However, these events were less frequent in the uninfected group.
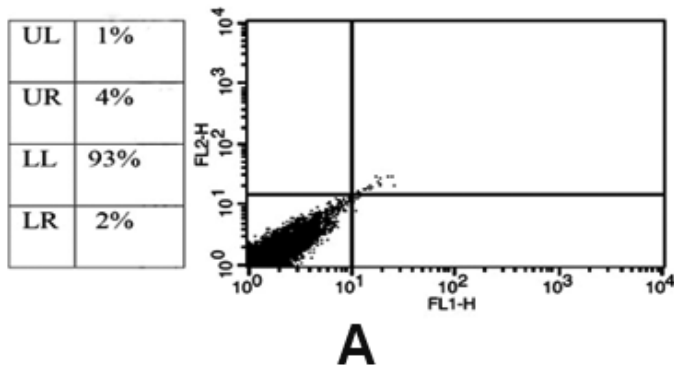
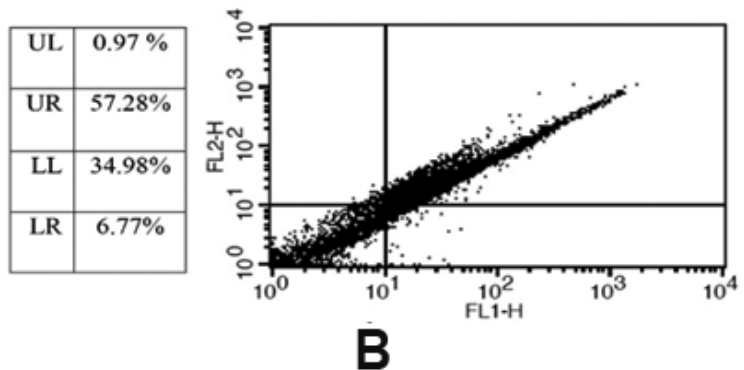
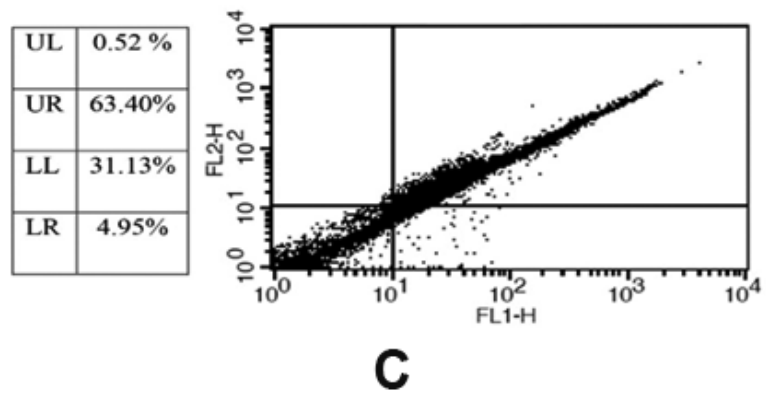

Figure 2. Flow cytometry analysis of fibroblast following treatment with supernatant of infected macrophage and uninfected macrophage extract $100 \mu \mathrm{g} / \mathrm{dL}$ and after labeling with annexin-V and PI (Propidium Iodide). Lower Right region (LR) belongs to apoptotic cells (annexin-positive) and Upper Left region (UL) belongs to necrotic cells (PI-positive). Upper Right region (UR) belongs to banded cell region with annexin and PI, and Lower Left region (LL) belongs to survived cells. FL2 is propidium iodide and FL1 is annexin V. The Figure A belongs to control, the Figure B is for macrophage + supernatant, and the Figure C is for fibroblast cells. 
Macrophages are part of the innate immune system cells. The consequences of the effects of innate immune responses in humans exposed to certain types of pollution are not known, and in this regard, two different scenarios can be presented. A rapid inflammatory response can sometimes enable the host to control the infection until the immune response arrives. However, rapid and robust responses to intrinsic immunity may alone, or by reinforcing the effects of acquired responses, in some cases lead to the development and intensification of the complications of the disease. Examples of such fact include increasing the level of IL-12 production in other protozoal diseases, such as malaria, or the use of this cytokine, as an immunotherapy agent in cancers. In immunohistochemical studies, increased levels of this cytokine have led to complete improvement in cancer in the tested mice.

Nevertheless, their effect on other cells can lead to the stimulation of secondary cytokine production, which could have irreparable pathophysiological effects. ${ }^{28-31}$ On the other hand, nitric oxide is one of the elements released from activated macrophages, which is considered as a free radical and is highly toxic and destructive, just like other free radicals. ${ }^{32}$ Macrophages are activated by Lipopolysaccharide (LPS) and it seems that the presence and activation of high levels of macrophages in the culture medium in the uninfected group may lead to secrete high levels of IL-12, and therefore it could be one of the reasons for increased mortality in the uninfected group.

However, the inhibition of IL-12 production at the onset of the parasite into the macrophage is one of the essential parasitic escape mechanisms from immune responses. When the parasite infects the macrophage, it does not allow macrophage to be activated. More importantly, the parasite continuously inhibits the release of cytokines such as IL-12 from infected cells and prevents them from stimulating such cytokines through several pathways. By the suppression of these cytokines and their stimulation pathways, the parasite begins to multiply until the cells break down and infect other surrounding cells, resulting in a complete parasite infection without the attention of the immune system. ${ }^{33,34}$ Surprisingly, as noted above, the introduction of the parasite into the macrophage does not permit macrophages to be activated. As a result, the passive macrophage also loses its ability to produce free radicals such as IL-12; this difference is seen between the uninfected and infected group. So it is expected that, unlike the uninfected group, which produces large amounts of free radicals, the other agents, which are released due to $L$. primary parasite infection, lead to the destruction of skin tissue. ${ }^{35-37}$

\section{Conclusions}

In the end, the destruction of human fibroblasts in the medium through both infected and uninfected groups was evident. Because macrophages do not have such harmful effects on skin cells in the normal state of the body, and on the other hand, the infected macrophages are inactive and cannot produce IL-12 and free radicals, such as nitrite oxide, it seems that what leads to tissue destruction due to infection with the L. major is the response of the macrophage to the presence of the parasite. In our current study, we did not fully explore which elements were released from macrophage infected with L. major and compared their levels with healthy macrophages. Therefore, the nature and mode of the postulated mediators remain to be explored in future studies.

\section{References}

1. Monge-Maillo B, López-Vélez R. Therapeutic options for old world cutaneous leishmaniasis and new world cutaneous and mucocutaneous leishmaniasis, Drugs 2013;73:1889-1920. doi:10.1007/s40265-013-0132-1.

2. Ghodratollah S, Hushang R, Mojtaba M, et al. Identification of Leishmania species by kinetoplast DNA-polymerase chain reaction for the first time in Khaf district, Khorasan-e-Razavi province, Iran. Trop Parasitol 2015;5:50. doi:10.4103/22295070.145587.

3. Kuramochi T, Hatabu T, Ito M, et al. Non-ulcerative cutaneous lesion in immunodeficient mice with Leishmania amazonensis infection. Parasitol Int 1999;48:47-53. doi:10.1016/S13835769(98)00040-3.

4. Xin L, Wanderley JL, Wang Y, et al. The magnitude of CD4+ T-cell activation rather than TCR diversity determines the outcome of Leishmania infection in mice. Parasite Immunol 2011;33:170-80. doi:10.1111/j.1365-3024.2010.01268.x.

5. Watanabe Y, Hamaguchi-Tsuru E, Morimoto N, et al. IL-5Induced Eosinophils Suppress the Growth of Leishmania amazonensis In Vivo and Kill Promastigotes In Vitro in Response to Either IL-4 or IFN- $\gamma$. DNA Cell Biol 2004;23:412-8. doi:10.1089/1044549041474805.

6. Soong L. Subversion and Utilization of Host Innate Defense by Leishmania amazonensis. Front Immunol 2012;3. doi:10.3389/fimmu.2012.00058.

7. Verhagen J, Akdis M, Traidlhoffmann C, et al. Absence of Tregulatory cell expression and function in atopic dermatitis skin, J Allergy Clin Immunol 2006;117:176-83. doi:10.1016/j.jaci.2005.10.040.

8. Trautmann A, Akdis M, Kleemann D, et al. T cell-mediated Fas-induced keratinocyte apoptosis plays a key pathogenetic role in eczematous dermatitis. J Clin Invest 2000;106:25-35. doi:10.1172/JCI9199.

9. Farley SM, Dotson AD, Purdy DE, et al. Fas Ligand Elicits a Caspase-Independent Proinflammatory Response in Human Keratinocytes: Implications for Dermatitis. J Invest Dermatol 2006;126.2438-51. doi:10.1038/sj.jid.5700477.

10. Lavrik I. Death receptor signaling. J Cell Sci 2005;118:265-7. doi:10.1242/jes.01610.

11. Guan DW, Ohshima T, Kondo T. Immunohistochemical study on Fas and Fas ligand in skin wound healing. Histochem J 2000;32:85-91.

12. Eidsmo L, Nylen S, Khamesipour A, et al. The Contribution of the Fas/FasL Apoptotic Pathway in Ulcer Formation during Leishmania major-Induced Cutaneous Leishmaniasis. Am J Pathol 2005;166:1099-108. doi:10.1016/S0002-9440(10) 62330-9.

13. Rethi B, Eidsmo L. FasL and TRAIL signaling in the skin during cutaneous leishmaniasis - implications for tissue immunopathology and infectious control. Front Immunol 2012;3:163. doi:10.3389/fimmu.2012.00163.

14. Eidsmo L, Fluur C, Rethi B, et al. FasL and TRAIL Induce Epidermal Apoptosis and Skin Ulceration Upon Exposure to Leishmania major. Am J Pathol 2007;170:227-39. doi:10.2353/ajpath.2007.060068.

15. Rahimnejad M, Derakhshanfar S, Zhong W. Biomaterials and tissue engineering for scar management in wound care. Burn Trauma 2017;5:4. doi:10.1186/s41038-017-0069-9.

16. Sakthianandeswaren A, Elso CM, Simpson K, et al. The wound repair response controls outcome to cutaneous leishma- 
niasis. Proc Natl Acad Sci 2005;102:15551-6. doi:10.1073/ pnas.0505630102.

17. von Stebut E. Cutaneous Leishmania infection: progress in pathogenesis research and experimental therapy. Exp Dermatol 2007;16:340-6. doi:10.1111/j.1600-0625.2007.00554.x.

18. Sakthianandeswaren A, Curtis JM, Elso C, et al. Fine Mapping of Leishmania major Susceptibility Locus $1 \mathrm{mr} 2$ and Evidence of a Role for Fli1 in Disease and Wound Healing. Infect Immun 2010;78:2734-44. doi:10.1128/IAI.00126-10.

19. Khanjani FA, Jafroodi S. Leishmania major in vitro and in vivo. Int Arch Heal Sci 2015;2;69-74.

20. Lucas M, Stuart LM, Zhang A, et al. Requirements for Apoptotic Cell Contact in Regulation of Macrophage Responses. J Immunol 2006;177:4047-54. doi:10.4049/jimmunol.177.6.4047.

21. Faulkner L, Patel M, Brickell PM, Katz DR. The role of the Fgr tyrosine kinase in the control of the adhesive properties of U937 monoblastoid cells and their derivatives. Immunology 1997;92:519-26.

22. Novais FO, Santiago RC, Bafica A, et al. Neutrophils and Macrophages Cooperate in Host Resistance against Leishmania braziliensis Infection. J Immunol 2009;183:808898. doi:10.4049/jimmunol.0803720.

23. Arab-Bafrani Z, Saberi A, Javad M, et al. Gold Nanoparticle and Mean Inactivation Dose of Human Intestinal Colon Cancer HT-29 Cells. Jundishapur J Nat Pharm Prod 2015;10:10-13. doi:10.17795/jjnpp-29153.

24. Abbasian M, Mousavi E, Khalili M, Arab-Bafrani Z. Using of keratin substrate for enrichment of HT29 colorectal cancer stem-like cells. J Biomed Mater Res Part B Appl Biomater 2019;107:1264-71. doi:10.1002/jbm.b.34219.

25. Abbasian M, Baharlouei A, Arab-Bafrani Z, Lightfoot DA. Combination of gold nanoparticles with low-LET irradiation: an approach to enhance DNA DSB induction in HT29 colorectal cancer stem-like cells. J Cancer Res Clin Oncol 2019;145:97-107. doi:10.1007/s00432-018-2769-3.

26. Mosser DM, Edwards JP. Exploring the full spectrum of macrophage activation. Nat Rev Immunol 2008;8:958-69. doi: $10.1038 /$ nri2448.

27. de Menezes JP, Saraiva EM, da Rocha-Azevedo B. The site of the bite: Leishmania interaction with macrophages, neutrophils and the extracellular matrix in the dermis. Parasit Vectors 2016;9:264. doi:10.1186/s13071-016-1540-3.

28. Arango Duque G, Descoteaux A. Macrophage Cytokines: Involvement in Immunity and Infectious Diseases. Front Immunol 2014;5. doi:10.3389/fimmu.2014.00491.

29. Chen L, He Z, et al. Antitumor Effect of Malaria Parasite Infection in a Murine Lewis Lung Cancer Model through Induction of Innate and Adaptive Immunity. PLoS One 2011;6:e24407. doi:10.1371/journal.pone.0024407.

30. Lin WW, Karin M. A cytokine-mediated link between innate immunity, inflammation, and cancer. J Clin Invest 2007;117: 1175-83. doi:10.1172/JCI31537.

31. Weiss JM, Subleski JJ, Wigginton JM, Wiltrout RH. Immunotherapy of cancer by IL-12-based cytokine combinations. Expert Opin Biol Ther 2007;7:1705-21. doi:10.1517/ 14712598.7.11.1705.

32. Vodovotz Y, Bogdan C, Paik J, et al. Mechanisms of suppression of macrophage nitric oxide release by transforming growth factor beta. J Exp Med 1993;178:605-13.

33. Martinez FO, Gordon S,. The M1 and M2 paradigm of macrophage activation: time for reassessment. F1000Prime Rep 2014;6:3. doi:10.12703/P6-13.

34. Schuster S, Hartley MA, Tacchini-Cottier F, Ronet C. A scoring method to standardize lesion monitoring following intradermal infection of Leishmania parasites in the murine ear. Front Cell Infect Microbiol 2014;4. doi:10.3389/ fcimb.2014.00067.

35. Eren RO, Reverte M, Rossi M, et al. Mammalian Innate Immune Response to a Leishmania -Resident RNA Virus Increases Macrophage Survival to Promote Parasite Persistence. Cell Host Microbe 2016;20:318-28. doi:10.1016/j.chom.2016.08.001.

36. Caljon G, De Muylder G, Durnez L, et al. Alice in microbes' land: adaptations and counter-adaptations of vector-borne parasitic protozoa and their hosts, FEMS Microbiol Rev 2016;40:664-85. doi:10.1093/femsre/fuw018.

37. Gurung P, Kanneganti TD. Immune responses against protozoan parasites: a focus on the emerging role of Nod-like receptors. Cell Mol Life Sci 2016;73:3035-51. doi:10.1007/s00018016-2212-3. 